\title{
A parameter to quantify the degree of genetic mixing among individuals in hybrid populations
}

\author{
ST Kalinowski and JH Powell
}

Hybridization between genetically distinct taxa is a complex evolutionary process. One challenge to studying hybrid populations is quantifying the degree to which non-native genes have become evenly mixed among individuals in the population. In this paper, we present a variance-based parameter, $m_{d}$, that measures the degree to which non-native genes are evenly distributed among individuals in a population. The parameter has a minimum value of 0 for populations in which individuals from multiple taxa are present but have not interbred, and a maximum value of 1 for populations in which all individuals have the same amount of non-native ancestry. A recurrence equation showed that relatively few generations of random mating are required for $m_{d}$ to approach 1 (indicating a well-mixed population), and that $m_{d}$ is independent of initial amounts of non-native ancestry. The parameter is mathematically equivalent to $F_{S T}$ and we show how existing formulae for $F_{S T}$ can be used to estimate $m_{d}$ when diagnostic loci are available. Computer simulations showed this estimator to have very little bias for realistic amounts of data. Heredity (2015) 114, 249-254; doi:10.1038/hdy.2014.93; published online 12 November 2014

\section{INTRODUCTION}

Hybridization between genetically distinct taxa is a complex ecological process that has important implications for a diverse array of population genetic questions. For example, medical geneticists study admixed human populations to identify and map genes causing diseases (for example, Mao et al., 2007; Cheng et al., 2010; Winkler et al., 2010). Ecological geneticists study admixed populations to understand how outbreeding affects survival and reproduction (for example, Hogg et al., 2006; Johnson et al., 2010; Vander Wal et al., 2012). Conservation geneticists study admixed populations to manage the spread of introgressive hybridization (for example, Rhymer and Simberloff, 1996; Hedrick, 2009; Muhlfeld et al., 2009). Agricultural geneticists and environmental activists monitor wild populations to detect genes from genetically modified organisms (for example, Watrud et al., 2004; Piñeyro-Nelson et al., 2009; Zapiola and Mallory-Smith, 2012). And finally, evolutionary biologists study hybrid zones to better understand how selection, gene flow and mate choice shape the genetic structure of natural populations (for example, Barton and Hewitt, 1985).

Hybridization often begins when non-native individuals enter a population and mate with individuals from a native taxon. At this point, the population may have a 'bimodal' distribution of genotypes (Harrison and Bogdanowicz, 1997). Such a population might consist of mostly genetically 'pure' individuals of both taxa, and, potentially, a few hybrid individuals. For some taxa, this will be as far as hybridization proceeds (for example, Steeves et al., 2010). However, if hybrids are fertile and pre-zygotic isolating mechanisms are weak, hybridization may continue until all individuals in the population are hybrids (Rhymer et al., 1994). At this point, ecologists sometimes call the population a 'hybrid swarm' (for example, Allendorf et al., 2001). If interbreeding continues, the distribution of non-native genes among individuals in the population will become 'unimodal' and will eventually approach the point in which all individuals have the same amount of non-native genes - and the mixing can be viewed as complete.

Genetic data are often used to quantify the amount of non-native genes in admixed populations and the degree to which these genes have become mixed in the population. The analysis of such data is relatively straightforward when there are fixed genetic differences between the taxa (for example, Rhymer et al., 1994), and sophisticated methods are available to study hybridization when fixed genetic differences between taxa are not present (for example, Pritchard et al., 2000; Anderson and Thompson, 2002; Hey, 2010). Therefore, it is usually relatively straightforward to use genetic data to estimate the ancestry of each individual in a hybrid population.

One challenge to interpreting such data is the potentially complex distribution of different levels of hybridity among individuals in the population. As discussed above, the amount of non-native genes present in individuals sometimes varies widely among the individuals in a population. Quantifying this variability is relevant to many analyses, but there is no widely accepted way to do this. A common practice is to report the total proportion, $P$, of non-native genes in a population and present a graph showing the distribution of non-native genes among all individuals (for example, Pertoldi et al., 2010). This approach conveys a lot of information about the distribution of non-native genes among individuals in a population, but makes it difficult to compare the degree of genetic mixing among different populations.

Vernesi et al. (2003) developed a parameter, which they called the 'true hybridization index' (THI), that solves this problem by quantifying how well the genes of multiple taxa are mixed in a population. THI has a range of $0-1$, with 0 indicating that no mixing has occurred 
(all individuals are genetically pure), and 1 indicating that the population is thoroughly mixed (all individuals have the same amount of ancestry from each contributing taxon).

The purpose of this present investigation is to extend Vernesi et al. (2003) work in several ways. First, we show how their genetic mixing parameter (which we call the 'degree of genetic mixing') can be derived in a simple, biologically meaningful way. This new definition allows us to show how the amount of genetic mixing in a population is related to the amount of gametic disequilibrium present in a population, and to show how the parameter increases in a randomly mating population. We also present a nearly unbiased formula for estimating this parameter when diagnostic loci are available and discuss how best to estimate the parameter when diagnostic loci are not available.

\section{METHODS AND RESULTS}

\section{A genetic mixing parameter, $\boldsymbol{m}_{d}$}

We seek to derive a parameter (We use 'parameter' to refer to a numeric characteristic of an entire population and 'statistic' to refer to a quantity calculated from a sample (Everitt and Skrondal, 2010)) that quantifies the degree of genetic mixing among taxa in a population and has a range of 0 to 1 . We will derive such a parameter here and compare it to the parameter THI of Vernesi et al. (2003) in the Discussion.

A parameter quantifying the degree of genetic mixing among taxa can be derived as follows. As above, let $P$ represent the overall proportion of non-native genes in a population. Furthermore, let $P_{i}$ represent the proportion of non-native genes in the genome of the $i$ th individual. Finally, let $N$ represent the total number of individuals in the population. With this notation, $P=\frac{1}{N} \sum_{i} P_{i}$. The variance of $P_{i}$, $\operatorname{Var}\left(P_{i}\right)$, serves as a useful measure of how well mixed native and nonnative genes are in the population. If the two taxa have interbred for a long time, all individuals in the population will have similar values of $P_{i}$, and $\operatorname{Var}\left(P_{i}\right)$ will be low. $\operatorname{Var}\left(P_{i}\right)$ will take a minimum value of zero when all individuals in the population have exactly the same amount of non-native ancestry. On the other hand, if there has not been extensive interbreeding between the taxa present in the population, $\operatorname{Var}\left(P_{i}\right)$ will be high. $\operatorname{Var}\left(P_{i}\right)$ will take a maximum value when all individuals in the population are genetically pure members of either taxa $\left(P_{i}\right.$ for every individual is either 0 or 1$)$. The variance of $P_{i}$ for this case is $P(1-P)$, the variance of a Bernoulli random variable. Given this, we propose a measure, which we will call $m_{d}$, of how well mixed non-native genes are in a population

$$
m_{d}=1-\frac{\operatorname{Var}\left(P_{i}\right)}{P(1-P)}
$$

This parameter is equivalent to THI as defined by Vernesi et al. (2003) (see below), but we call it the 'degree of genetic mixing' in a population because we do not believe it is appropriate to call any parameter describing the amount of hybridization in a population the 'true hybrid index'. There are lots of reasonable ways of quantifying hybridization in a population, and, therefore, no single 'true' index. This parameter has a minimum value of 0 , which occurs when a population consists of individuals from two species that have not yet interbred, that is, $\operatorname{Var}\left(P_{i}\right)=P(1-P)$. This parameter has a maximum value of 1.0, which occurs when all the individuals in the population have the same amount of non-native ancestry. Note that $m_{d}$ is undefined if $P$ equals 0 or 1 . This is appropriate, as it does not make sense to quantify how genetically well mixed a population is when there are genes from only one taxon in the population.
Our parameter can also be defined for hybrid populations having more than two taxa. Let $P_{j}$ represent the proportion of the genes in the population that belong to the $j$ th taxa and let $P_{i j}$ represent the proportion of genes in the $i$ th individual that are from the $j$ th taxa. With this notation, $m_{d}$ is equal to

$$
m_{d}=1-\frac{\sum_{j} \operatorname{Var}\left(P_{j i}\right)}{\sum_{j} P_{j}\left(1-P_{j}\right)}
$$

\section{Genetic mixing and gametic disequilibrium}

It is well known that recently hybridized populations have high amounts of gametic disequilibrium (even at unlinked loci), and that this disequilibrium declines with time. This suggests that there may be a relationship between $m_{d}$ and the amount of gametic disequilibrium in a population. This, indeed, is the case. Let $D$ represent the parametric amount of gametic disequilibrium present at a pair of loci in a hybrid population, and let $\bar{D}$ represent the average value of $D$ across all the pairs of loci in the population. Barton and Gale (1993); Equation 2b have shown that for populations in Hardy-Weinberg equilibrium

$$
\operatorname{Var}\left(P_{i}\right)=\frac{1}{2} \bar{D}
$$

If we divide both sides of Equation 3 by $P(1-P)$, we obtain

$$
\frac{\operatorname{Var}\left(P_{i}\right)}{P(1-P)}=\frac{1}{2} \bar{D}^{\prime}
$$

where $D^{\prime}$ is a popular standardized measure of gametic disequilibrium that has a maximum value of 1.0 (Lewontin, 1964; Hedrick, 2011). Combining Equations 1 and 4 shows the relationship between $m_{d}$ and $D^{\prime}$

$$
m_{d}=1-\frac{1}{2} \bar{D}^{\prime}
$$

This is a very useful result for two reasons. First, it relates the degree of mixing in a population to the amount of gametic disequilibrium present in the population, a quantity frequently estimated in genetic samples. Second, it allows us to make quantitative statements about how quickly genes in a population will mix when there is random mating.

\section{Genetic mixing in randomly mating populations}

Interpreting empirical estimates of $m_{d}$ would be easier if we knew how quickly it could increase in simple evolutionary scenarios, for example, if there was random mating in a population. The relationship between $m_{d}$ and $D^{\prime}$ makes it easy to make some simple statements about the behavior of $m_{d}$ in cases like this. For example, it is well known that $D$ and $D^{\prime}$ for unlinked loci decrease by a factor of 0.5 in a randomly mating population (for example, Hedrick, 2011). This fact tells that if we use unlinked loci to estimate the ancestry of individuals, $1-m_{d}$ will decrease by a factor of 0.5 every generation of random mating. Therefore, if a population begins with genetically pure individuals from two taxa $\left(m_{d}=0\right)$, and the individuals in the population mate randomly for $t$ generations, $m_{d}$ at generation $t, m_{d}{ }^{(\mathrm{t})}$, will equal

$$
m_{d}{ }^{(t)}=1-\left(\frac{1}{2}\right)^{t}
$$

For the first three generations of random mating, $m_{d}$ will equal $1 / 2$, $3 / 4$ and $7 / 8$ (Figure 1). After five generations of random mating, $m_{d}$ will be $\sim 0.97$, and mixing will be nearly complete. This relationship assumes that the population begins with genetically pure 
individuals from two taxa, but is independent of the proportion of non-native individuals that enter the population.

\section{Estimation}

We will discuss estimation of $m_{d}$ in two contexts: cases in which diagnostic loci are available and cases in which diagnostic loci are not available.

\section{Estimation using diagnostic loci}

Estimating $m_{d}$ using diagnostic loci is facilitated by noting the term $\operatorname{Var}\left(P_{i}\right) / P(1-P)$ in Equation 1 is mathematically equivalent to Wright's $F_{S T}$ (Wright, 1951), or more specifically, $F_{S T}$ for one locus with two alleles. The only difference between $m_{d}$ and $F_{S T}$ is that $P_{i}$ in Equation 1 refers to the frequency of alleles in an individual whereas $P_{i}$ in Wright's (1951) definition of $F_{S T}$ refers to the frequency of alleles in a population. The similarity is not coincidental. $F_{S T}$ quantifies how allele frequencies vary among populations; our parameter, $m_{d}$, quantifies how allele frequencies vary among individuals. The mathematical equivalence between $m_{d}$ and $F_{S T}$ allows us to use the well-developed literature on $F_{S T}$ to estimate $m_{d}$ (see below).

If diagnostic loci are available to unambiguously discriminate between native and non-native alleles, standard methods for estimating $F_{S T}$ can be used to estimate $m_{d}$. For example, Weir and Cockerham's $\hat{\theta}$ (1984) can be used to produce an estimate of $m_{d}, \hat{m}_{d}$

$$
\hat{m}_{d}=1-\hat{\theta}
$$

Weir and co-workers have presented a few alternative methods for estimating $\hat{\theta}$ (Weir and Cockerham, 1984; Weir and Hill, 2002; Weir, 2010). The method most appropriate for the application here is the estimator designed to compare gene pools in randomly mating populations (Weir and Cockerham, 1984, unlabeled equation at the top of page 1363; Weir and Hill, 2002, Equation 5). Using our notation, this estimator is calculated as

$$
\hat{\theta}=\frac{\frac{1}{N_{\mathrm{s}}-1} \sum_{i} n_{i}\left(\hat{P}_{i}-P\right)^{2}-\frac{1}{\sum_{i}\left(n_{i}-1\right)} \sum_{i} n_{i} \hat{P}_{i}\left(1-\hat{P}_{i}\right)}{\frac{1}{N_{\mathrm{s}}-1} \sum_{i} n_{i}\left(\hat{P}_{i}-P\right)^{2}+\frac{(\bar{n}-1)}{\sum_{i}\left(n_{i}-1\right)} \sum_{i} n_{i} \hat{P}_{i}\left(1-\hat{P}_{i}\right)}
$$

where $N_{\mathrm{s}}$ is the number of individuals sampled from a hybrid population, $n_{i}$ is the number of amplified alleles in individual $i, \hat{P}_{i}$ is the estimated proportion of non-native alleles in the genome of individual $i$, and $\bar{n}$ is the average number of alleles amplified in each genotyped individual

$$
\bar{n}=\frac{1}{N_{\mathrm{s}}-1}\left(\sum_{i=1}^{n} n_{i}-\frac{\sum_{i} n_{i}^{2}}{\sum_{i} n_{i}}\right)
$$

These equations, as noted above, are only applicable for loci having diagnostic alleles.

$\theta$ can also be calculated using software designed for estimating $F_{S T}$ (for example; GENEPOP; Rousset, 2008). Doing this requires reorganizing the data so that each individual in the sample is represented as a population and the diagnostic alleles from all loci are pooled into a single locus. Appendix shows how this can be done to create a GENEPOP file (Rousset, 2008).

Weir and Cockerham's (1984) $\hat{\theta}$ is essentially unbiased when used to estimate $F_{S T}$. However, $\hat{\theta}$ may not be as unbiased when used to estimate $m_{d}$. Weir and Cockerham's formula for $\hat{\theta}$ (Equation 8 in our paper) assumes alleles sampled from an individual are random and independent draws from a gene pool (which, in our application, is an individual's genome). With this assumption, the estimated proportion of non-native ancestry in the $i$ th individual, $\hat{P}_{i}$, will be binomially
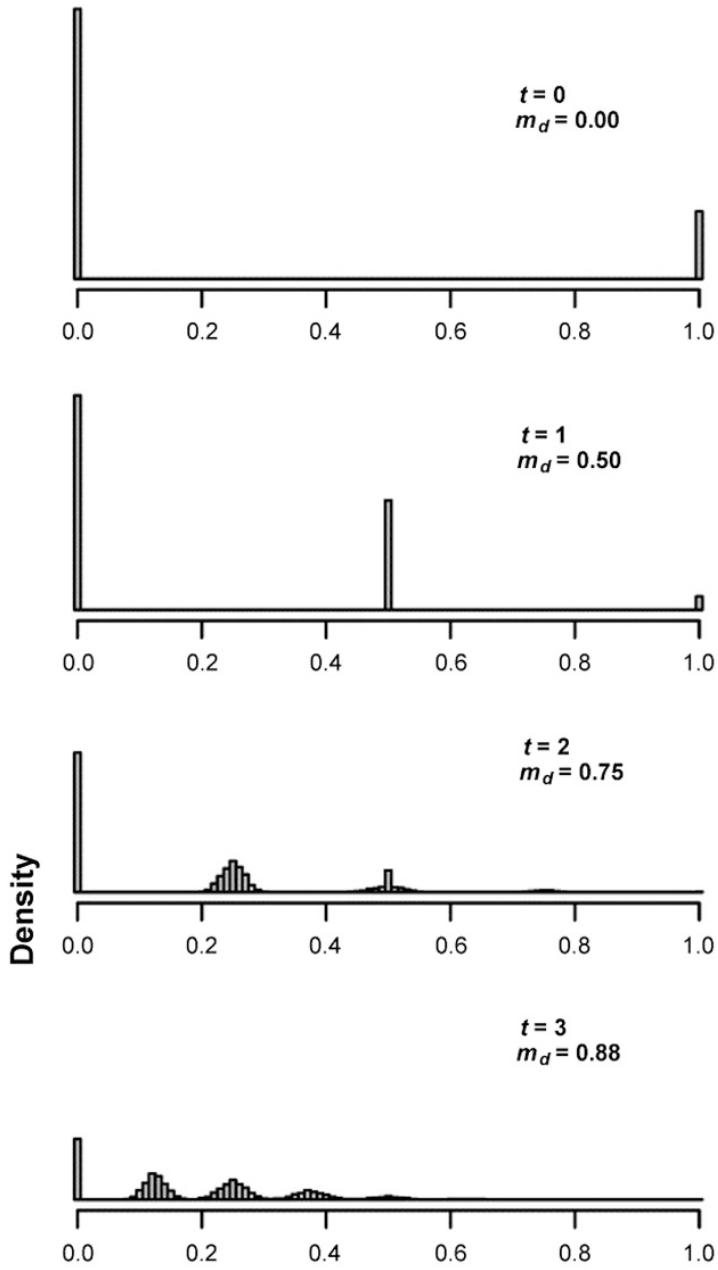

$t=4$
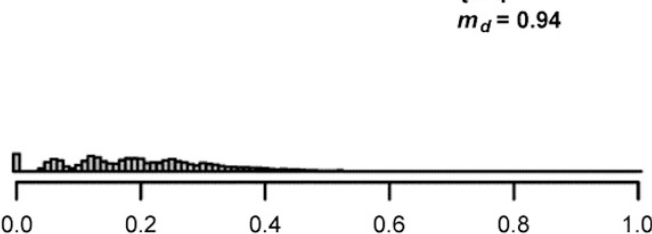

$\boldsymbol{t}=\mathbf{5}$

$m_{d}=0.97$

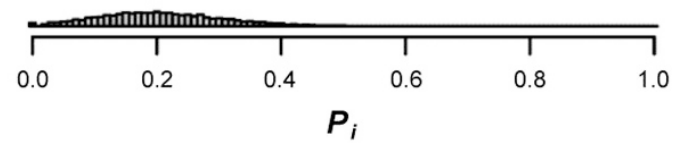

Figure 1 The distribution of hybridity coefficients $\left(P_{j}\right)$ among individuals in a randomly mating populations over five generations $(t=0-5)$. Individuals with $P_{i}$ equal to 0 or 1 are genetically pure members of alternative taxa.

distributed. This will not be true when there is gametic disequilibrium in a population (for example, in the early generations of hybridization). When there is gametic disequilibrium in a population, the alleles present in diploid genotypes are not always going to be independent. This is easily seen by considering an $F_{1}$ individual (that is, an individual having one parent from each hybridizing taxon). In an $F_{1}$ 
Table 1 Statistical bias of $\hat{m}_{d}$ calculated from Equation 6 and 9 estimated from computer simulations for samples of varying sizes $\left(N_{\text {Loci, }}, N_{\text {Individuals }}\right)$ from a population of 200 individuals after $t$ generations of random mating (see text for details)

\begin{tabular}{|c|c|c|c|c|c|c|}
\hline \multirow{3}{*}{$\mathrm{N}_{\text {Loci }}$} & \multicolumn{3}{|c|}{$t=2: m_{d}=0.75$} & \multicolumn{3}{|c|}{$t=5: m_{d}=0.97$} \\
\hline & \multicolumn{3}{|c|}{$\mathrm{N}_{\text {Individuals }}$} & \multicolumn{3}{|c|}{$\mathrm{N}_{\text {Individuals }}$} \\
\hline & 10 & 20 & 50 & 10 & 20 & 50 \\
\hline 8 & 0.0330 & 0.0326 & 0.0325 & 0.0065 & 0.0062 & 0.0059 \\
\hline 16 & 0.0154 & 0.0150 & 0.0150 & 0.0036 & 0.0034 & 0.0032 \\
\hline 48 & 0.0043 & 0.0039 & 0.0039 & 0.0015 & 0.0015 & 0.0014 \\
\hline 96 & 0.0016 & 0.0012 & 0.0012 & 0.0010 & 0.0010 & 0.0010 \\
\hline
\end{tabular}

Note that the bias was positive in all the cases; this indicates that the estimated values of $m_{d}$ tended to be higher than the parametric value.

individual, every locus will be heterozygous and $P_{i}$ will equal 0.5 . Because each locus is heterozygous, $\hat{P}_{i}$ will also equal 0.5 -no matter how few or many loci are genotyped. In other words, when an $F_{1}$ individual is genotyped, there will be no sampling error in $\hat{P}_{i}$. Equation 8 assumes binomial sampling error, so terms in Equation 8 intended to eliminate sampling bias will not work as intended. This could result in a negative estimate of $\operatorname{Var}\left(P_{i}\right) / P(1-P)$, which would produce an estimate of $m_{d}$ that is $>1.0$. This is equivalent to an estimate of $F_{S T}$ being < zero.

We used computer simulations to estimate how much bias there was in the estimates of $m_{d}$ calculated from Equation 7 and 8 for realistic amounts of data. We simulated populations of 50, 200 and 2000 randomly mating individuals that were founded with $20 \%$ nonnative individuals. We modeled these populations after cutthroat trout (Oncorhynchus clarkii) - a species which frequently hybridizes with non-native rainbow trout (O. mykiss) - and assumed the ratio of the genetic effective population size, $N_{\mathrm{e}}$, to census size, $N$, was 0.23 (Finger et al., 2011). We achieved this ratio of $N_{\mathrm{e}}$ to $N$ by varying the reproductive success according to the method of Anderson (2001). For each individual, 52 chromosomal arms were simulated with 10 equally spaced, species-specific diagnostic di-allelic loci. Recombination was allowed to occur once per chromosomal arm per generation (Danzmann et al., 2005). In each generation we calculated the true degree of mixing, $m_{d}$, for each population. Then we drew 1000 simulated samples of $10,20,50$ or 100 individuals with genotypes at 8 , 16, 48 and 96 loci. The bias in estimates of $m_{d}$ was calculated by comparing the average estimate of $m_{d}$ with the parametric value for the populations, that is, bias $=$ average $\left(\hat{m}_{d}\right)-m_{d}$.

The simulations were performed using a program written by us in $\mathrm{R}$ (R Development Core Team, 2008).

The computer simulations showed that there was only a modest amount of bias in the first few generations of mating, and this bias was becoming negligible by the fifth generation (Table 1). In all cases, estimates of $m_{d}$ tended to be slightly higher than the parametric value (that is, the bias was positive). As expected, the amount of bias was greatest in small samples, and for populations in the early stages of hybridization. The smallest samples we examined had 10 individuals genotyped at eight diagnostic loci. Even with these small samples, the bias observed in the second generation (0.0330), was only $4.4 \%$ of the parametric value $(0.75)$. When the number of diagnostic loci was increased to 16 , the bias was reduced to just over $2 \%$. The bias present in the estimates of $m_{d}$ for populations that had five generations of random mating $\left(m_{d}=0.97\right)$ was much lower. Even for the smallest samples, it was only $0.6 \%$ of the parametric value.

\section{Estimation using non-diagnostic loci}

When diagnostic loci are not available, $m_{d}$ can be estimated by estimating the ancestry of each individual in a sample, and then inserting these estimates into Equations 1 or 2, (depending on the number of taxa involved). There are a variety of methods for estimating the ancestry $\left(P_{i}\right)$ of hybrid individuals using loci that do not have diagnostic alleles (for example, Pritchard et al., 2000; Anderson and Thompson, 2002). The computer programs STRUCTURE and NEWHYBRIDS have been popular for this type of analysis. The accuracy of estimates of $m_{d}$ obtained from $P_{i}$ 's calculated by these programs will depend on how well the $P_{i}^{\prime} s$ are estimated. $m_{d}$ is calculated from the variance of $P_{i}$, so if there is a bias in this variance, there will be a bias in estimates of $m_{d}$. Little is known about the error structure of STRUCTURE or similar analytic approaches, but it is likely that these errors will be affected by the amount of genetic differentiation among the taxa being studied, the number of loci genotyped and the amount of genetic variation at those loci.

We performed a series of computer simulations to explore how estimation error in $P_{i}^{\prime} s$ affected estimates of $m_{d}$. A systematic investigation of the error structure present in STRUCTURE or NEWHYBRIDS is beyond the scope of this investigation, so we examined how three plausible, generic models of estimation error affected estimates of $m_{d}$. In all simulations, we assumed there were two hybridizing taxa. As above, we simulated populations of 50, 200 or 2000 individuals and kept track of the parametric values of $P_{i}$ for each individual in the population. Estimates of $P_{i}$ were obtained in the simulation by assuming one of the three statistical models of estimation error. The first model assumed that the ancestry of one of the taxa was always underestimated by a constant amount. The second model assumed that estimates of the ancestry of two taxa were biased towards 0.5 by a constant proportion. The third model assumed that estimation error was normally distributed. We tested different magnitudes of estimation error for each model and ran 10000 simulations for each case to quantify the bias in estimates of $m_{d}$.

The simulations showed that the bias in estimates of $m_{d}$ was a function of how well the ancestry of each individual, $P_{i}$, was estimated (results not shown). Good estimates of $P_{i}$ produced good estimates of $m_{d}$. There did not appear to be any thresholds affecting how estimation error in $P_{i}$ was propagated to estimates of $m_{d}$.

\section{DISCUSSION}

We have defined and derived a parameter, $m_{d}$, that quantifies the amount of genetic mixing of native and non-native genes in a hybrid population. The parameter that we developed is related to both $F_{S T}$ and the average amount of pairwise gametic disequilibrium in a population. The value of this parameter will rapidly approach 1.0 in randomly mating populations; after five generations of random mating, $m_{d}$ will be $\sim 0.97$. Computer simulations showed that when diagnostic loci are available, one of Weir and Cockerham's (1984) estimators of $F_{S T}$ did a very nice job of estimating $m_{d}$. If diagnostic loci are not available, $m_{d}$ can be estimated by inserting the estimated ancestry of each individual into Equations 1 or 2,.

The genetic mixing parameter described here may be thought of as an alternative version of the 'true hybridization index' (THI) parameter of Vernesi et al. (2003). Both parameters have a range of 0 to 1.0 and both parameters quantify as to how well genes are mixed in a population. In fact, $m_{d}$ and THI are mathematically equivalent. The main difference between $m_{d}$ and THI is how the parameters are 
defined. $m_{d}$ is defined as a variance of the amount of non-native genes across individuals in a population. As noted above, this variance will be zero when the population is genetically well mixed and all individuals have the same amount of non-native ancestry. THI is defined as the average variance of genetic ancestry within individuals. This is explained as follows. The first step in calculating THI is calculating the variance of ancestry, $V_{i}$, within each individual in the sample. If $d$ taxa are hybridizing, and we use our notation, the variance of nonnative genes in the $i$ th individual is $V_{i}=\frac{\sum_{j}\left(P_{i j}-\frac{1}{d}\right)^{2}}{d}$. Notice that this variance measures how close the native and non-native ancestries of an individual are to $1 / d$. This quantity, somewhat surprisingly, is related to the amount of mixing in a population. If all the individuals in a population are genetically pure, the proportion of native and nonnatives genes in the $i$ th individual, $P_{i j}$, will be 0 or 1 , and the values of $V_{i}$ for every individual will be relatively high. On the other hand, if all individuals in a population have the same amount of non-native genes, the values of $P_{i j}$ will be closer to $1 / d$ and the values of $V_{i}$ will be smaller. This is the principle THI uses to quantify the amount of mixing in a population. More specifically, THI is calculated by taking the average of $V_{i}$ across individuals and then standardized to account for the minimum and maximum values of this average (given the amount of non-native genes in the population). This calculation produces a quantity with the same value as $m_{d} . m_{d}$ has the advantage of a simple, easy-to-interpret definition. This is important because it allowed us to relate $m_{d}$ to the amount of gametic disequilibrium in a population, identify how $m_{d}$ changed in randomly mating populations, and estimate $m_{d}$ using formulae for $F_{S T}$.

In randomly mating populations, $m_{d}$ will rapidly approach 1.0 at a rate specified by Equation 6. There are, however, many plausible reasons as to why natural populations will behave differently. For example, if mating between the two taxa is not random, genetic mixing may proceed more slowly. Alternatively, if hybrid individuals have a lower evolutionary fitness, mixing will be slower. Mixing will also appear to be slower if non-native individuals continuously enter a population. And, finally, the rate of mixing will be affected by the genotypes of the first individuals to enter a population. If these individuals have mixed ancestry, $m_{d}$ will be higher than if the first immigrants were genetically pure non-natives.

A few comments regarding the definition of a 'hybrid swarm' may be useful, as several definitions are present in the literature. Rhymer and Simberloff (1996) defined a hybrid swarm as a population containing individuals with various degrees of non-native ancestry. Allendorf et al. (2001) used a slightly different definition; they specified that all individuals in a hybrid swarm must be hybrids. Finally, Allendorf and Leary (1988) provided the most strict definition; they defined a hybrid swarm as a population in which all individuals in the population have the same amount of non-native ancestry (that is, $m_{d}=1$ ). All of these definitions may be useful, but in some circumstances it may be more useful to quantify how well mixed non-native genes are in a population rather than to classify a population as being a hybrid swarm or not. This is especially likely to be true as the number of loci used to study hybridization increases. Genomic data are expected to have very high power to detect slight amounts of variation in ancestry. Therefore, when genomic data are available, it may not be useful to define a hybrid swarm as a population in which all individuals have the same amount of nonnative ancestry.

We conclude this paper with a discussion of what it means for genes in a hybrid population to be 'mixed.' We have assumed throughout this paper that native and non-native genes are well mixed when all the individuals in the population have the same amount of non-native ancestry. This criterion should be useful for studying populations that are in the early stages of hybridization or have stable distributions of hybrid genotypes. Other mixing criteria and parameters may be more informative for examining populations that have been hybridizing for a long time. In particular, if populations have been hybridizing for a long time, it may be more useful to quantify how thoroughly recombination has broken up and shuffled the genomes of native and non-native taxa into small chromosomal segments (for example, vonHoldt et al., 2011 and references within). When genomic data are available, such an approach can be used to date the timing of admixture (Tang et al., 2006). If such data are not available, and a population is in the early stages of admixture, the mixing degree parameter presented in this paper should be informative.

\section{DATA ARCHIVING}

There were no data to deposit.

\section{CONFLICT OF INTEREST}

The authors declare no conflict of interest.

\section{ACKNOWLEDGEMENTS}

We thank reviewers for thoughtful comments that improved this manuscript. Funding for this project was provided by the National Science Foundation (DEB 0717456 to STK and DGE 1049562 to JHP) and the United States Geological Service.

Allendorf FW, Leary RB, Spruell P, Wenburg JK (2001). The problems with hybrids: setting Conservation guidelines. Trends Ecol Evol 16: 613-622.

Allendorf FW, Leary RF (1988). Conservation and distribution of genetic variation in a poltypic species, the cutthroat trout. Conserv Biol 2: 170-184.

Anderson EC (2001). Monte Carlo Methods for Inference in Population Genetic Models. University of Washington: Seattle, WA, USA, $195 \mathrm{p}$.

Anderson EC, Thompson EA (2002). A model-based method for identifying species hybrids using multilocus genetic data. Genetics 160: 1217-1229.

Barton NH, Gale KS (1993). Genetic analysis of hybrid zones. In: Harrison RG (ed). Hybrid Zones and the Evolutionary Process. Oxford University Press, New York, NY, USA, pp 13-45.

Barton NH, Hewitt M (1985). Analysis of hybrid zones. Annual Review of Ecology and Systematic 16: 113-148.

Cheng C-Y, Reich D, Coresh J, Boerwinkle E, Patterson N, Li M et al. (2010). Admixture mapping of obesity-related traits in African Americans: the Atherosclerosis Risk in Communities (ARIC) Study. Obesity 18: 563-572.

Danzmann RG, Cairnet M, Davidson WS, Ferguson MM, Gharbi K, Guyomard R et al. (2005). A comparative analysis of the rainbow trout genome with 2 other species of fish (Arctic charr and Atlantic salmon) within the tetraploid derivative Salmonidae family (subfamily: Salmoninae). Genome 48: 1037-1051.

Everitt BS, Skrondal A (2010). The Cambridge Dictionary of Statistics. Cambridge University Press, Cambridge, UK.

Finger AJ, Anderson EC, Stephens MR, May BP (2011). Application of a method or estimating effective population size and admixture using diagnostic single nucleotide polymorphisms (SNPS): implications for conservation of threatened Paiute cutthroat trout (Oncorhynchus clarkii seleniris) in Silver King Creek. Can J Fish Aquat Sci 68: 1369-1386

Harrison RG, Bogdanowicz SM (1997). Patterns of variation and linkage disequilibrium in a field cricket hybrid zone. Evolution 493-505.

Hedrick PW (2011). Genetics of Populations. Jones and Bartlett Publishers: Sudbury, MA, USA.

Hedrick PW (2009). Conservation genetics and North American bison (Bison bison). $J$ Hered 100: 411-420.

Hey J (2010). Isolation with migration models for more than two populations. Mol Biol Evol 27: 905-920.

Hogg JT, Forbes SH, Steele BM, Luikart G (2006). Genetic rescue of an insular population of large mammals. Proc Biol Sci 273: 1491-1499.

Johnson WE, Onorato DP, Roelke ME, Land ED, Cunningham M, Belden RC et al. (2010). The genetic restoration of the Florida panther. Science 329: 1641-1645.

Lewontin RC (1964). The interaction of selection and linkage. I. General considerations, heterotic models. Genetics 49: 49-67.

Mao X, Bigham AW, Mei R, Gutierrez G, Weiss KM, Brutsaert TD et al. (2007). A genomewide admixture mapping panel for hispanic/latino populations. Am J Hum Genet 80: 1171-1178. 
Muhlfeld CC, Kalinowski ST, McMahon TE, Painter S, Leary RF, Taper ML et al. (2009). Hybridization reduces fitness of cutthroat trout in the wild. Biol Lett 5: 328-331.

Pertoldi C, Tokarska M, Wójcik J.M, Kawako A, Randi E, Kristensen TN et al. (2010). Phylogenetic relationships among the European and American bison and seven cattle breeds reconstructed using the BovineSNP50 Illumina Genotyping BeadChip. Acta Theriol 55: 97-108.

Piñeyro-Nelson A, Van Herwaarden J, Perales HR, Serratos-Hernandez JA, Rangel A, Hufford MB et al. (2009). Transgenes in Mexican maize: molecular evidence and methodological considerations for GMO detection in landrace populations. Mol Ecol 18: 750-761.

Pritchard JK, Stephens M, Donnelly P (2000). Inference of population structure using multilocus genotype data. Genetics 155: 945-959.

Rhymer JM, Williams MJ, Braun MJ (1994). Mitochondrial analysis of gene flow between New Zealand mallards (Anas platyrhynchos) and grey ducks (A. supreciliosa). Auk 111. 970-978.

Rhymer JM, Simberloff D (1996). Extinction by hybridization and introgression. Annu Rev Ecol Syst 27: 83-109.

Rousset F (2008). Genepop'007: a complete reimplementation of the genepop software for Windows and Linux. Mol Ecol Resour 8: 103-106.

R Development Core Team (2008). R: A language and environment for statistical computing. $\mathrm{R}$ Foundation for Statistical Computing: Vienna, Austria. ISBN. 3-900051-07-0, URL http://www.R-project.org.

Steeves TE, Maloney RF, Hale ML, Tylianakis JM, Gemmel NJ (2010). Genetic analyses reveal hybridization but no hybrid swarm in one of the world's rarest birds. $\mathrm{Mol} E \mathrm{Co} / 19$ : 5090-5100.

Tang H, Coram M, Wang P, Zhu X, Risch N (2006). Reconstructing genetic ancestry blocks in admixed individuals. Am J Hum Genet 79: 1-12.

Vander Wal E, Garant D, Festa-Bianchet M, Pelletier F (2012). Evolutionary rescue in vertebrates: evidence, applications and uncertainty. Phil Trans $R$ Soc $B 368$ 20120090.

Vernesi C, Crestanello B, Pecchioli E, Tartari D., Caramelli D, Hauffe H et al. (2003). The genetic impact of demographic decline and reintroduction in the wild boar (Sus scrofa): a microsatellite analysis. Mol Ecol 12: 585-595.

vonHoldt BM, Pollinger JP, Earl DA, Knowles JC, Boyko AR, Boyko AR et al. (2011). A genome-wide perspective on the evolutionary history of enigmatic wolf-like canids. Genome Resour 21: 1294-1305.

Watrud LS, Lee EH, Fairbrother A, Burdick C, Reichman JR, Bollman M et al. (2004). Evidence for landscape-level, pollen-mediated gene flow from genetically modified creeping bentgrass with CP4 EPSPS as a marker. Proc Natl Acad Sci USA 40: $14533-14538$.

Weir BS (2010). Genetic Data Analysis III. Sinauer Associates, Inc: Sunderland, MA, USA Weir BS, Cockerham CC (1984). Estimating F-statistics for the analysis of population structure. Evolution 38: 1358-1370.

Weir BS, Hill WG (2002). Estimating F-statistics. Annu Rev Genet 36: 721-750.

Winkler CA, Nelson GW, Smith MW (2010). Admixture mapping comes of age. Annu Rev Genomics Hum Genet 11: 65-89.

Wright S (1951). The genetical structure of populations. Ann Eugen 15: 323-353.

Zapiola ML, Mallory-Smith CA (2012). Crossing the divide: gene flow produces intergeneric hybrid in feral transgenic creeping bentgrass population. $\mathrm{Mol} E \mathrm{CO} / 21$ : 4672-4680.

\section{APPENDIX}

GENEPOP files with genotypes of standard format and reformatted to estimate $\operatorname{Var}\left(P_{i}\right) / P(1-P)$. As an example, let us consider a sample of four fish, in which: two fish are native fish with no non-native genes, one fish is an F1 hybrid, and the last fish is a pure nonnative fish.

Here is a sample data file for these four individuals with diploid genotypes at three loci.

Sample GENEPOP data in normal format

Diagnostic Locus1, Diagnostic Locus2, Diagnostic

Locus3

POP

$\begin{array}{llll}\text { Ind1, } & 11 & 11 & 11 \\ \text { Ind2, } & 11 & 11 & 11 \\ \text { Ind3, } & 12 & 12 & 12 \\ \text { Ind4, } & 22 & 22 & 22\end{array}$

The data file above can be reformatted to estimate $\operatorname{Var}\left(P_{i}\right) / P(1-P)$ using GENEPOP (see below). Note that each individual in the original sample is considered a 'population' in the reformatted GENEPOP file. Note also the data is reformatted to be haploid.

\section{Sample GENEPOP data reformatted to calculate Var} $\left(\mathrm{P}_{\mathrm{i}}\right) /[\mathrm{P}(\mathrm{PP})]$

Diagnostic Loci

POP

Ind1Locusla, $\quad 01$

Ind1Locus1b, 01

Ind1Locus2a, 01

Ind1Locus2b, 01

Ind1Locus3a, $\quad 01$

Ind1Locus3b, 01

POP

Ind2Locusla, $\quad 01$

Ind2Locus1b, 01

Ind2Locus2a, 01

Ind2Locus2b, 01

Ind2Locus3a, $\quad 01$

Ind2Locus3b, 01

POP

Ind3Locusla, $\quad 01$

Ind3Locus1b, 02

Ind3Locus2a, 01

Ind3Locus2b, 02

Ind3Locus3a, 01

Ind3Locus3b, 02

POP

Ind4Locusla, $\quad 02$

Ind4Locus1b, 02

Ind4Locus2a, 02

Ind4Locus2b, 02

Ind4Locus3a, 02

Ind4Locus3b, 02

In this example, the parametric value of $m_{d}$ is equal to 0.2667 . An estimate calculated from GENEPOP's estimate of $\operatorname{Var}\left(P_{i}\right) / P(1-P)$ is equal to 0.2571 . 